PROCEEDINGS OF THE

AMERICAN MATHEMATICAL SOCIETY

Volume 48, Number 2, April 1975

\title{
A NOTE ON A COROLLARY OF SARD'S THEOREM
}

\author{
JOHN C. WELLS
}

ABSTRACT. A corollary of Sard's theorem is the following:

Corollary. Let $f: K \rightarrow R^{n}$ be a smooth (i.e. $f \in C^{k}, k \geq 1$ ) map defined on a compact subset $K$ of $R^{n}$. Let $C=\left\{y \mid f^{-1}(y)\right.$ is infinite $\}$. Then the Lebesgue measure of $C$ is zero.

The purpose of this note is to show that a similar version of this theorem holds for Lipschitz functions.

Theorem. Let $f: K \rightarrow R^{n}$ with $f$ Lipschitz, $K$ a measurable subset of $R^{n}$ and $m(K)$ the Lebesgue measure of $K$, less than $\infty$. Let $C=\left\{y \mid f^{-1}(y)\right.$ is infinite\}. Then $m(C)=0$.

The proof of the theorem will require two lemmas.

Lemma 1. Let $f: K \rightarrow R^{m}$ with $K$ a compact subset of $R^{n}$ and $f a$ continuous function. Then there exists $K^{\prime} \subset K$ with $f\left(K^{\prime}\right)=f(K),\left.f\right|_{K^{\prime}}$ one to one and $K^{\prime}$ a Borel set.

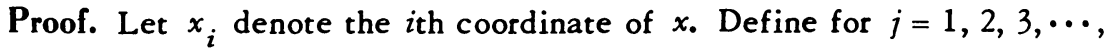
$A_{j}=\left\{x \mid x \in K\right.$ and there exists $y \in K$ with $y_{1} \leq x_{1}-1 / j$ and $f(y)=f(x)$ or $y \in K$ with $y_{1}=x_{1}, y_{2} \leq x_{2}-1 / j$ and $f(y)=f(x)$ or $\cdots$ or $y \in K$ with $y_{1}$ $=x_{1}, \cdots, y_{n-1}=x_{n-1}, y_{n} \leq x_{n}-1 / j$ and $\left.f(y)=f(x)\right\}$. Since $A_{j}$ is compact for each $j, K^{\prime}=K-\bigcup_{j} A_{j}$ is Borel. If $x \in f(K)$ observe that $y$ defined by

$$
y_{1}=\operatorname{Inf}\left\{z_{1} \mid f(z)=f(x)\right\}, \cdots, y_{n}=\operatorname{Inf}\left\{z_{n} \mid z_{1}=y_{1}, \cdots, z_{n-1}=y_{n-1}, f(z)=f(x)\right\}
$$

is not contained in $A_{j}$ for any $j$ and also $f(y)=f(x)$. Thus $f\left(K^{\prime}\right)=f(K)$. Clearly $\left.f\right|_{K}$, is one to one.

Lemma 2. Let $f: K \rightarrow R^{m}$ be a continuous map from $K$ a measurable subset of $R^{n}$. Then there exist $K^{\prime}, K^{\prime \prime}$ with $K^{\prime \prime} \subset K^{\prime} \subset K, K^{\prime \prime}$ and $K^{\prime}$ Borel sets, $\left.f\right|_{K^{\prime \prime}}$ one to one, $m\left(K-K^{\prime}\right)=0$ and $f\left(K^{\prime \prime}\right)=f\left(K^{\prime}\right)$.

Proof. There exists an $F_{\sigma}$ set $K^{\prime}$, with $K^{\prime} \subset K$ and $m\left(K-K^{\prime}\right)=0$, and we can write $K^{\prime}=\bigcup_{n} K_{n}$ where $K_{n}$ is compact for each $n$. By Lemma 1

Received by the editors April 3, 1974 and, in revised form, June 26, 1974. AMS (MOS) subject classifications (1970). Primary 26A63, 28A75. 
there are Borel sets $K_{n}^{\prime} \subset K_{n}$ with $f\left(K_{n}^{\prime}\right)=f\left(K_{n}\right)$ and $\left.f\right|_{K_{n}^{\prime}}$ one tn one for each $n$. Define recursively $D_{1}=K_{1}^{\prime}, \cdots, D_{k}=D_{k-1} \cup\left(K_{k}^{\prime}-f^{-1}\left(f\left(D_{k-1}\right)\right)\right)$. Then $K^{\prime \prime}=\bigcup_{k} D_{k}$ is Borel, $f\left(K^{\prime \prime}\right)=f\left(K^{\prime}\right)$ and $\left.f\right|_{K^{\prime \prime}}$ is one to one.

Proof of Theorem. Suppose $m(C)=a>0$. Let $A_{1}=f^{-1}(C)$ and find by Lemma $2 N_{1}^{\prime \prime} \subset N_{1}^{\prime} \subset A_{1}$ with $N_{1}^{\prime \prime}$ and $N_{1}^{\prime}$ Borel, $m\left(A_{1}-N_{1}^{\prime}\right)=0,\left.f\right|_{N_{1}^{\prime \prime}}$ one to one and $f\left(N_{1}^{\prime \prime}\right)=f\left(N_{1}^{\prime}\right)$. Since $f$ is Lipschitz, $m\left(f\left(A_{1}-N_{1}^{\prime}\right)\right)=0$. Therefore $m\left(f\left(N_{1}^{\prime \prime}\right)\right)=a$. Now $f\left(A_{1}-N_{1}^{\prime \prime}\right)=C$ by the definition of $C$. Thus for $k=2,3, \cdots$ by letting $A_{k}=A_{k-1}-N_{k-1}^{\prime \prime}$ we can repeat the above argument to find $N_{k}^{\prime \prime} \subset N_{k}^{\prime} \subset A_{k}$ with $\left.f\right|_{N_{k}^{\prime \prime}}$ one to one, $f\left(N_{k}^{\prime \prime}\right)=f\left(N_{k}^{\prime}\right)$ and $m\left(f\left(N_{k}^{\prime \prime}\right)\right)=a$. Suppose that $L$ is the Lipschitz constant for $f$; then $m\left(N_{k}^{\prime \prime}\right) \geq a / L$ for each $K$. But since $N_{k}^{\prime \prime} \cap N_{k}^{\prime}=\varnothing$ for $k=k^{\prime}$, this implies $m(K)=\infty$, a contradiction.

Remarks. The requirement that $f$ be Lipschitz in the theorem cannot be weakened to a requirement of continuity. To show this let $f$ be the first coordinate of a continuous map of the unit interval $I$ onto the unit square $I^{2}$ (i.e. a space-filling curve). Then $f^{-1}(y)$ is infinite for each $y \in I$.

On the other hand, for $n=1$ absolute continuity will suffice as the following argument shows. Suppose $f$ is a real valued a.c. function on 1 . Then $f$ is differentiable except on a set $A_{1}$ of measure zero. $m\left(f\left(A_{1}\right)\right)=0$ since $f$ is a.c. Let $A_{2}=\left\{x \mid f^{\prime}(x)=0\right\}$ and let $\eta$ be an arbitrary positive number. For each $x \in A_{2}$ there are arbitrarily small intervals containing $x$ such that $|f(y)-f(x)| \leq|y-x|$ when $y$ is in the intervals. Thus the collection $\mathcal{F}$ of all such intervals with $x$ varying over $A_{2}$ is a Vitale covering of $A_{2}$, so there is a disjoint collection $\left\{I_{n}\right\} \subset \mathcal{F}^{\prime}$ with $m\left(A_{2}-\bigcup_{n} I_{n}\right)=0$. But then $m\left(f\left(A_{2}\right)\right) \leq m\left(f\left(A_{2}-\bigcup_{n} I_{n}\right)\right)+m\left(f\left(\bigcup_{n} I_{n}\right)\right) \leq 0+2 \eta \cdot \sum_{n}$ length $\left(I_{n}\right) \leq 2 \eta$.

Since $\eta$ is arbitrary, $m\left(f\left(A_{2}\right)\right)=0$. Finally, if $y \in f(I)-f\left(A_{1}\right)-f\left(A_{2}\right)$ then $f^{-1}(y)$ must consist of isolated points and hence be finite. Thus $m\left\{y \mid f^{-1}(y)\right.$ is infinite $\}=0$.

\section{REFERENCES}

1. John Milnor, Topology from the differential viewpoint, Univ. Press of Virginia, Charlottesville, Va., 1965. MR 37 \#2239.

2. A. Sard, The measure of the critical values of differential maps, Bull. Amer. Math. Soc. 48 (1942), 883-890. MR 4, 153.

DEPARTMENT OF MATHEMATICS, CALIFORNIA STATE UNIVERSITY, NORTHRIDGE, CALIFORNIA 91324 